\title{
Dietary Acid Load and Incident Chronic Kidney Disease: Results from the ARIC Study
}

\author{
Casey M. Rebholz ${ }^{a, b}$ Josef Coresh ${ }^{a-c}$ Morgan E. Grams $^{a, b, d}$ Lyn M. Steffen $^{e}$ \\ Cheryl A.M. Anderson ${ }^{b, f}$ Lawrence J. Appel ${ }^{a-c}$ Deidra C. Crews ${ }^{a, d}$
}

aWelch Center for Prevention, Epidemiology, and Clinical Research, Johns Hopkins University, ${ }^{b}$ Department of Epidemiology, Johns Hopkins Bloomberg School of Public Health, 'Division of General Internal Medicine, Department of Medicine, Johns Hopkins School of Medicine, and d Division of Nephrology, Department of Medicine, Johns Hopkins School of Medicine,

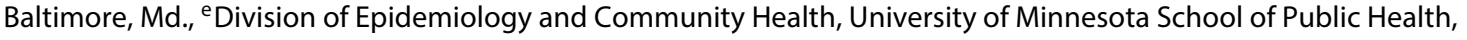
Minneapolis, Minn., and f Division of Preventive Medicine, Department of Family Medicine and Public Health, University of California San Diego School of Medicine, San Diego, Calif., USA

\section{Key Words}

Diet - Chronic kidney disease · Magnesium - Vegetable protein

\begin{abstract}
Background: Higher dietary acid load can result in metabolic acidosis and is associated with faster kidney disease progression in patients with chronic kidney disease (CKD). However, the relationship between dietary acid load and incident CKD has not been evaluated. Methods: We conducted prospective analyses of the Atherosclerosis Risk in Communities study participants without CKD at baseline (1987-1989, n = $15,055)$. Dietary acid load was estimated using the equation for potential renal acid load by Remer and Manz, incorporating dietary intake data from a food frequency questionnaire. Incident CKD was assessed from baseline through 2010 and defined as estimated glomerular filtration rate (eGFR) $<60$ $\mathrm{ml} / \mathrm{min} / 1.73 \mathrm{~m}^{2}$ accompanied by $25 \%$ eGFR decline, CKDrelated hospitalization or death or end-stage renal disease identified by linkage to the US Renal Data System registry. Results: In the overall study population, 55\% were female, $26 \%$ were African-American and mean age at baseline was 54 years. During a median follow-up of 21 years, there were
\end{abstract}

\section{KARGER}

E-Mail karger@karger.com

www.karger.com/ajn
2,351 (15.6\%) incident CKD cases. After adjusting for demographics (age, sex, race-center), established risk factors (diabetes status, hypertension status, overweight/obese status, smoking status, education level, physical activity), caloric intake and baseline eGFR, higher dietary acid load were associated with higher risk of incident CKD (hazard ratio [HR] for quartile 4 vs. $1: 1.13,95 \% \mathrm{Cl} 1.01-1.28, \mathrm{p}$ for trend $=0.02 ; \mathrm{HR}$ per interquartile range increase: $1.06,95 \% \mathrm{Cl} 1.00-1.11, \mathrm{p}=$ $0.04)$. Conclusion: Dietary acid load is associated with incident CKD in a population-based sample. These data suggest a potential avenue for CKD risk reduction through diet.

(c) 2016 S. Karger AG, Basel

\section{Introduction}

The optimal diet for preventing kidney disease onset and progression has not been identified. Dietary acid load or the balance between acid-inducing foods such as animal sources of protein and base-inducing foods such as fruits and vegetables may be injurious to the kidney. Higher dietary acid load can increase metabolic acidosis and thereby lead to increased risk of kidney disease progression $[1,2]$. The contemporary western diet is largely 
acid-inducing and has been posited to contribute to the increasing prevalence of chronic kidney disease (CKD) in the United States [3,4]. Of the available studies on dietary acid load, most of them included patients with prevalent CKD [5-8]. These studies suggest that higher dietary acid load can result in faster kidney disease progression in patients with CKD.

Given the growing prevalence of $\mathrm{CKD}$, prevention strategies are needed to reduce the number of individuals affected by this disease and its associated morbidities and cost $[4,9,10]$. Prior studies of dietary acid load have not evaluated incident CKD. If dietary acid load is associated with incident CKD, it would represent an opportunity for prevention among individuals with preserved kidney function.

The objective of the present study was to test the hypothesis that higher dietary acid load is associated with greater risk of incident CKD in a general population sample.

\section{Methods}

\section{Study Population and Design}

The Atherosclerosis Risk in Communities (ARIC) study is a community-based observational study of 15,792 middle-aged (45-64 years) adults enrolled in 1987-1989 from 4 US communities: (1) Forsyth County, North Carolina; (2) Jackson, Mississippi; (3) suburbs of Minneapolis, Minnesota and (4) Washington County, Maryland [11]. In the present prospective analysis, participants were excluded for the following reasons: (1) missing diet data or implausible caloric intake defined as $<500$ or $>3,500 \mathrm{kcal} /$ day for women and $<700$ or $>4,500 \mathrm{kcal} /$ day for men $(\mathrm{n}=364)$, (2) prevalent CKD at baseline as defined below $(n=328)$ or (3) neither Caucasian nor African-American race $(\mathrm{n}=45$; fig. 1$)$. After these exclusions, the analytic sample size was 15,055 (95\% of original cohort). At each of the 4 participating study centers, an Institutional Review Board reviewed and approved the study protocol. Study participants provided informed consent at each study visit.

\section{Assessment of Dietary Intake}

Usual dietary intake was assessed using a semi-quantitative, 66item food frequency questionnaire that was modified from the Willett questionnaire [12]. This questionnaire demonstrated high reproducibility in a random sample of ARIC study participants $(\mathrm{n}=419)$ and was administered by trained interviewers at the baseline examination (study visit 1, 1986-1989) and at a follow-up examination (study visit 3, 1993-1995) [13]. To reduce within-individual variation and to better represent usual dietary intake, we analyzed the cumulative average diet [14]. That is, between baseline and study visit 3 , we used dietary intake data collected at baseline, and after study visit 3 , we used the mean of values from baseline and study visit 3 .

Participants reported how often they consumed each food item of a specified portion size on average during the last year in the

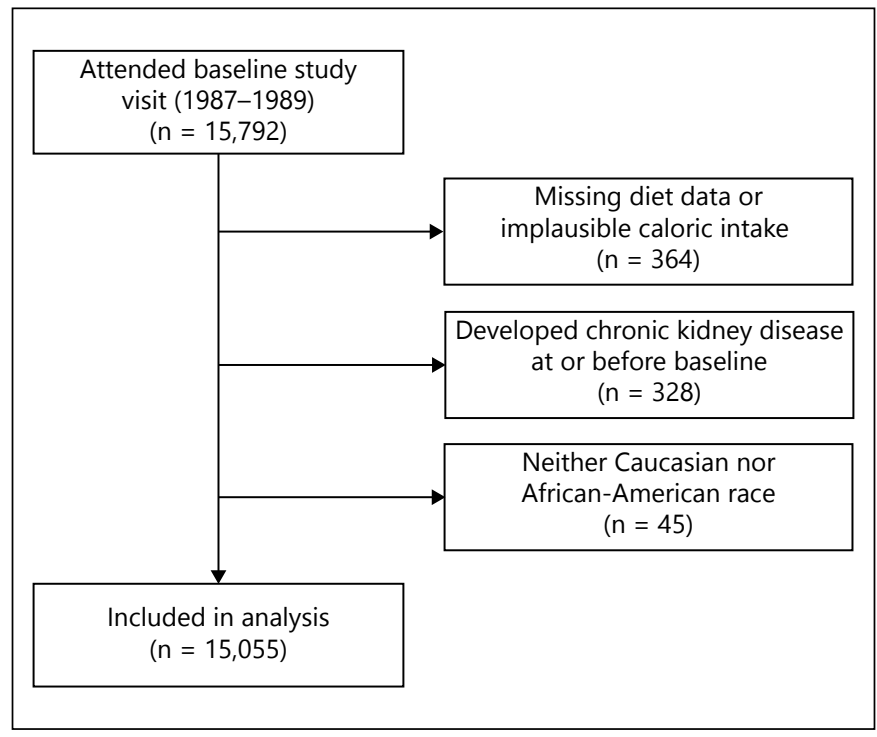

Fig. 1. Flow chart of study participant selection.

following categories: almost never, 1-3/month, 1/week, 2-4/week, 5-6/week, 1/day, 2-3/day, 4-6/day and >6/day. Daily intake of micro- and macronutrients was calculated by multiplying frequency of consumption and portion size of each food item by its nutritional content [15].

\section{Quantification of Dietary Acid Load}

Dietary acid load was estimated using the equation for potential renal acid load (PRAL) by Remer and Manz [16]: PRAL $=0.49$ $*$ protein $+0.037 *$ phosphorus $-0.021 *$ potassium $-0.026^{*}$ magnesium $-0.013 *$ calcium. As a sensitivity analysis, we estimated net endogenous acid production (NEAP), which is the ratio of protein to potassium intake and another means of evaluating dietary acid load: NEAP $=54.5 *($ protein/potassium $)-10.2$ [17]

\section{Definition of Incident CKD}

Incident CKD was a composite outcome defined as at least 1 of the 4 following criteria: (1) development of reduced kidney function (estimated glomerular filtration rate $[\mathrm{eGFR}]<60 \mathrm{ml} / \mathrm{min} / 1.73 \mathrm{~m}^{2}$ ) accompanied by $25 \%$ eGFR decline at any subsequent study visit relative to baseline, (2) International Classification of Diseases (ICD)-9/10 code for a hospitalization related to CKD stage $3+$ identified through active surveillance of the ARIC cohort, (3) ICD-9/10 code for a death related to CKD stage $3+$ identified through linkage to the National Death Index or (4) end-stage renal disease identified by linkage to the US Renal Data System (USRDS) registry. Development of CKD was assessed between baseline (visit 1, 19871989) and the end of follow-up for this analysis of the ARIC study (December 31, 2010). Combining data from study visits and intervening events captured by surveillance of hospitalizations, the $\mathrm{Na}$ tional Death Index and the USRDS registry mitigates the potential selection bias due to differential study visit attendance by kidney disease status. Although this definition differs slightly from clinical guidelines, it improves CKD ascertainment during periods of time between study visits and is appropriate for research studies [18]. 
We have previously validated this composite outcome by comparing it to medical chart review and it was shown to have high specificity $(96 \%)$ [19].

\section{Assessment of Covariates}

At baseline, a questionnaire was administered to study participants by trained interviewers to collect information on demographic characteristics (date of birth, sex, race/ethnicity), health behaviors (frequency, duration and intensity of physical activity; cigarette smoking), socioeconomic status (level of education) and health history (medication use, diagnosed diseases). Three measurements of blood pressure were taken by a trained technician after 5 min of rest using a random-zero sphygmomanometer, and the mean of the latter 2 measurements was used for analysis. Fasting blood samples were collected during the baseline examination. Serum glucose was measured by the modified hexokinase/glucose-6-phosphate dehydrogenase method, and serum creatinine was measured by the modified kinetic Jaffe method.

Hypertension was defined as systolic blood pressure $\geq 140 \mathrm{~mm}$ $\mathrm{Hg}$, diastolic blood pressure $\geq 90 \mathrm{~mm} \mathrm{Hg}$ or use of anti-hypertension medication in the preceding 2 weeks. Diabetes was defined as fasting glucose $\geq 126 \mathrm{mg} / \mathrm{dl}$, non-fasting glucose $\geq 200 \mathrm{mg} / \mathrm{dl}$, history of diagnosed diabetes or use of diabetes medication in the preceding 2 weeks. Body mass index was calculated as weight in kilograms divided by height in meters squared using measurements taken during the baseline examination. Overweight or obese status was defined as body mass index $\geq 25 \mathrm{~kg} / \mathrm{m}^{2}$. Kidney function was assessed using the 2009 Chronic Kidney Disease Epidemiology Collaboration (CKD-EPI) equation for eGFR based on serum creatinine [20].

\section{Statistical Analysis}

Baseline demographic, clinical and dietary factors were described according to quartile of dietary acid load using means, standard deviations and proportions. Spearman's rank correlation was used to assess the association between dietary acid load estimates at baseline and visit 3. Cox proportional hazards regression was used to estimate hazard ratios (HRs) and 95\% CIs for the association between estimates of dietary acid load at baseline (visit 1 , 1987-1989) and incident CKD during follow-up adjusted for age, sex, race-center, total caloric intake, diabetes status, hypertension status, overweight/obese status, smoking status, education level, physical activity and baseline kidney function. Kidney function (eGFR) was modeled as linear spline terms with a knot at $90 \mathrm{ml} /$ $\mathrm{min} / 1.73 \mathrm{~m}^{2}$. Given the different racial distribution at each of the 4 centers, we created an interaction variable for race $\times$ center. For CKD risk estimates as well as baseline characteristics, trend across quartiles was tested with linear regression using the median value within each quartile for continuous variables and with $\chi^{2}$ test for trend for categorical variables. Restricted cubic splines were used to present adjusted HRs for CKD by continuous dietary acid load estimates with knots at the 5 th, 35 th, 65 th and 95 th percentiles. For the graphical depiction of the splines, dietary acid load estimates were truncated at the 1st and 99th percentiles.

Relevant to our sensitivity analysis of dietary acid load estimates, Spearman's rank correlation was used to quantify the association between PRAL and NEAP. As another sensitivity analysis, analyses were repeated after dropping extreme values for dietary acid load (PRAL: $<-60$ and >60 mEq, NEAP: >110 mEq), and re- sults were similar to those from the main analysis. All analyses were performed using Stata statistical software version 14 (StataCorp LC, College Station, Tex., USA).

\section{Results}

In the overall ARIC study population, $55 \%$ were female, 26\% were African-American and mean age was 54 years. The median level (25th, 75th percentiles) of PRAL and NEAP, respectively, were $4.5(-3.2,12.1) \mathrm{mEq} /$ day and $48.0(40.1,56.9) \mathrm{mEq} /$ day. Baseline dietary acid load estimates were moderately correlated with dietary acid load estimates at visit 3 (PRAL: $\mathrm{r}=0.38, \mathrm{p}<0.001$; NEAP: $\mathrm{r}=0.39, \mathrm{p}<0.001)$.

At baseline, participants consuming higher levels of dietary acid load tended to be younger, AfricanAmerican, male, non-smokers, overweight or obese, less educated, less physically active and more likely to have diabetes and hypertension ( $p$ for all $<0.001$; table 1 ). There were small but statistically significant differences in mean baseline eGFR according to quartile of dietary acid load. Dietary intake of total protein, animal sources of protein and phosphorus were higher among those with higher levels of dietary acid load ( $p$ for all $<0.001$ ). The 2 estimates of dietary acid load - PRAL and NEAP - were highly correlated $(\mathrm{r}=0.95, \mathrm{p}<0.001)$.

During a median follow-up of 21 years, there were 2,351 (15.6\%) incident CKD cases. Higher dietary acid load was significantly associated with elevated CKD risk, even after adjusting for age, sex, race-center, total caloric intake, diabetes, hypertension, overweight/obese status, smoking, level of education, physical activity and baseline eGFR (table 2; fig. 2a). There was a statistically significant trend with higher levels of dietary acid load associated with higher risk of incident CKD (HR for quartile 4 vs. 1: $1.13,95 \%$ CI 1.01-1.28, $\mathrm{p}$ for trend $=0.02$ ). Each interquartile range (IQR) increase in dietary acid load was associated with $6 \%$ higher risk of incident CKD (HR 1.06, $95 \%$ CI $1.00-1.11, \mathrm{p}=0.04)$. In a sensitivity analysis using NEAP, findings were slightly stronger than those from the main analysis using PRAL (HR for quartile 4 vs. 1: $1.14,95 \%$ CI $1.01-1.28$, p for trend $=0.01$; HR per IQR increase: $1.08,95 \%$ CI 1.03-1.14, p = 0.004; fig. 2b).

Among the individual components of the dietary acid load estimates, higher dietary intake of magnesium (HR for quartile 4 vs. 1: $0.72,95 \%$ CI $0.60-0.85$, p for trend $<0.001$; HR per IQR: $0.87,95 \%$ CI $0.79-0.95, \mathrm{p}=0.002$ ) and vegetable sources of protein (HR for quartile 4 vs. 1: $0.72,95 \%$ CI $0.61-0.85$, p for trend $<0.001$; HR per IQR: 
Table 1. Baseline characteristics ${ }^{1}$ according to quartile of potential renal acid load

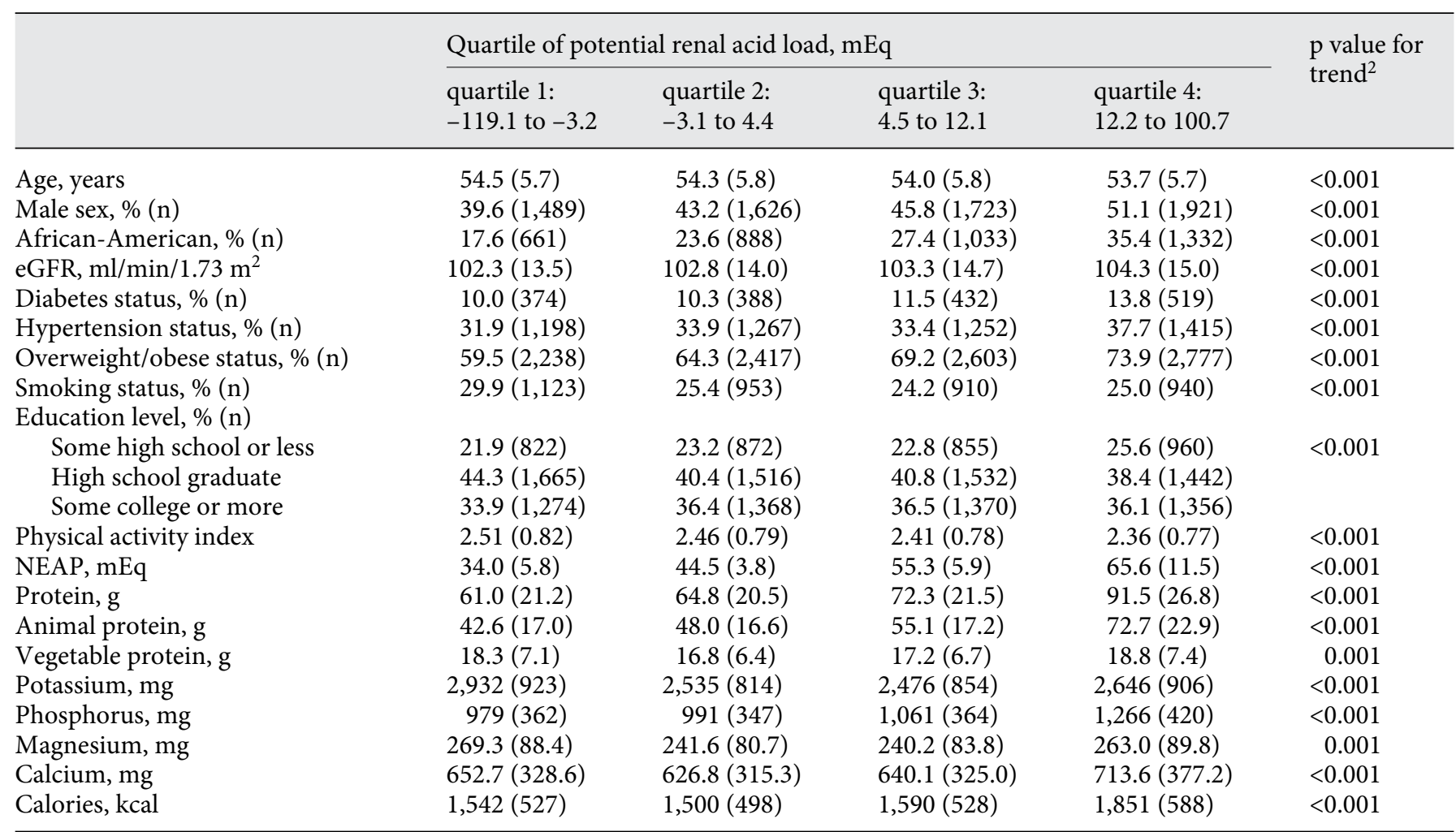

\footnotetext{
${ }^{1}$ Mean (SD) for continuous variables and $\%(\mathrm{n})$ for categorical variables.

2 Trend across quartiles was tested with linear regression using the median value within each quartile for continuous variables and with $\chi^{2}$ test for trend for categorical variables.
}

Table 2. Adjusted ${ }^{1}$ HRs (95\% CIs) for incident CKD by dietary factor

\begin{tabular}{|c|c|c|c|c|c|c|c|}
\hline & \multicolumn{4}{|c|}{ Quartile of dietary factor } & \multirow{2}{*}{$\begin{array}{l}\mathrm{p} \text { value for } \\
\text { trend }^{2}\end{array}$} & \multirow[t]{2}{*}{ Per IQR } & \multirow{2}{*}{$\begin{array}{l}\mathrm{p} \\
\text { value }\end{array}$} \\
\hline & quartile 1 & quartile 2 & quartile 3 & quartile 4 & & & \\
\hline NEAP & 1 (ref.) & $0.96(0.86-1.09)$ & $1.06(0.95-1.20)$ & $1.14(1.01-1.28)$ & 0.01 & $1.08(1.03-1.14)$ & 0.004 \\
\hline Protein & 1 (ref.) & $0.99(0.88-1.12)$ & $0.90(0.78-1.03)$ & $1.03(0.87-1.22)$ & 0.95 & $1.04(0.95-1.14)$ & 0.40 \\
\hline Animal protein & 1 (ref.) & $0.96(0.86-1.09)$ & $0.95(0.84-1.08)$ & $1.06(0.91-1.23)$ & 0.45 & $1.06(0.99-1.14)$ & 0.10 \\
\hline Phosphorus & 1 (ref.) & $0.95(0.84-1.07)$ & $0.88(0.77-1.01)$ & $0.81(0.68-0.96)$ & 0.01 & $0.97(0.89-1.07)$ & 0.57 \\
\hline Magnesium & 1 (ref.) & $0.90(0.80-1.02)$ & $0.80(0.70-0.92)$ & $0.72(0.60-0.85)$ & $<0.001$ & $0.87(0.79-0.95)$ & 0.002 \\
\hline Calcium & 1 (ref.) & $1.01(0.90-1.14)$ & $0.91(0.81-1.03)$ & $0.80(0.69-0.92)$ & 0.001 & $0.96(0.90-1.02)$ & 0.17 \\
\hline
\end{tabular}

${ }^{1}$ Adjusted for age, sex, race-center, total caloric intake, diabetes status, hypertension status, overweight/obese status, smoking status, education level, physical activity and baseline eGFR (modeled as linear spline terms with a knot at $90 \mathrm{ml} / \mathrm{min} / 1.73 \mathrm{~m}$ ).

2 Trend across quartiles was tested with linear regression using the median value within each quartile for continuous variables and with $\chi^{2}$ test for trend for categorical variables. 
Fig. 2. Adjusted* HRs (95\% CIs) for incident CKD according to dietary acid load estimated by PRAL (a) and NEAP (b). * Adjusted for age, sex, race-center, total caloric intake, diabetes status, hypertension status, overweight/obese status, smoking status, education level, physical activity and baseline eGFR (modeled as linear spline terms with a knot at $90 \mathrm{ml} / \mathrm{min} / 1.73$ $\mathrm{m}^{2}$ ). The shaded area represents the $95 \%$ CIs. Restricted cubic spline with knots at the 5 th, 35th, 65 th and 95 th percentiles and truncation at the 1st and 99th percentiles.

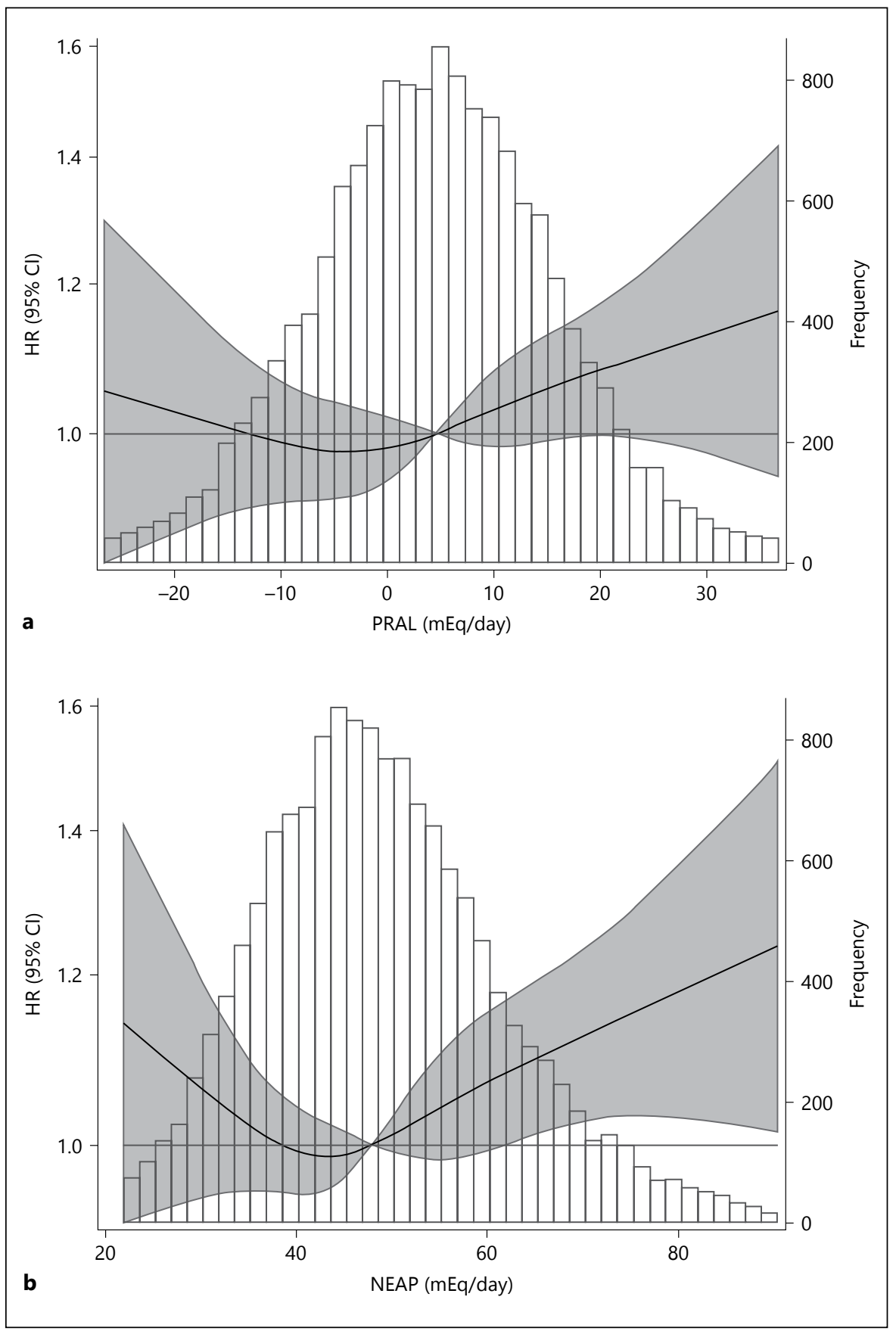

$0.89,95 \%$ CI $0.82-0.96, \mathrm{p}=0.004$ ) were strongly associated with a lower risk of CKD. In contrast with the protective association observed for vegetable sources of protein, total dietary protein and animal sources of protein were not significantly associated with CKD risk. Higher intake of phosphorus and calcium appeared to be associated with a reduced risk of CKD across quartiles ( $\mathrm{p}$ for trend $\leq 0.01$ ), but not in the continuous analysis $(\mathrm{p} \geq 0.17)$.

\section{Discussion}

In this large, population-based study of individuals without kidney disease, higher levels of dietary acid load were associated with higher risk of developing CKD over 21 years of follow-up independent of sociodemographic, clinical and lifestyle factors. This finding was consistent for 2 different estimates of dietary acid load based on self- 
reported dietary intake: PRAL and NEAP. Of the individual components of the dietary acid load estimates, higher intake of magnesium and vegetable sources of protein had the strongest protective association with CKD.

To the best of our knowledge, this is the first study to prospectively assess the relationship between dietary acid load and incident CKD. There are 3 recently published studies which lend further support to the concept of dietary acid load influencing kidney function. First, in a cross-sectional analysis using data from National Health and Nutrition Examination Survey (NHANES) 19992004, the highest versus lowest quintile of dietary acid load estimated by net acid excretion had 1.57 times ( $95 \%$ CI 1.20-2.05, $\mathrm{p}$ for trend $=0.04$ ) higher odds of albuminuria, but was not significantly associated with reduced eGFR or CKD stage defined according to both eGFR and albuminuria [21]. Additionally, among 1,486 individuals with eGFR $15-60 \mathrm{ml} / \mathrm{min} / 1.73 \mathrm{~m}^{2}$ in NHANES III, the highest tertile of net acid excretion was associated with a 3 -fold higher risk of end-stage renal disease [6]. Lastly, in 632 participants from the African American Study of Kidney Disease and Hypertension trial and cohort study with median GFR of $49 \mathrm{ml} / \mathrm{min} / 1.73 \mathrm{~m}^{2}$, higher levels of NEAP were significantly associated with faster decline in eGFR ( $p$ for trend $=0.01$ in adjusted analyses), but was not associated with incident end-stage renal disease over a median follow-up period of 8 years [5]. Our study extends these previous reports by documenting a novel finding of the association between dietary acid load and incident $\mathrm{CKD}$ and elucidating dietary factors (i.e., magnesium, vegetable sources of protein) that may be the primary drivers of this association.

Previous studies have demonstrated that diet can modify acid-base homeostasis which may in turn reduce the risk of disease progression in patients with kidney disease $[22,23]$. In an experimental study, individuals with stage 1 (eGFR $>90 \mathrm{ml} / \mathrm{min} / 1.73 \mathrm{~m}^{2}$ ) or stage 2 (eGFR 60$89 \mathrm{ml} / \mathrm{min} / 1.73 \mathrm{~m}^{2}$ ) CKD were assigned to either fruits and vegetables or sodium bicarbonate to modify dietary acid load or to a control group for 1 month [7]. Dietary acid load reduction by consumption of fruits and vegetables decreased kidney injury markers (urine concentrations of $\mathrm{N}$-acetyl $\beta$-D-glucosaminidase, albumin and transforming growth factor- $\beta$ ) among individuals with stage $2 \mathrm{CKD}$, but not for those with stage $1 \mathrm{CKD}$. Their findings for dietary acid reduction through fruit and vegetable consumption were comparable to oral sodium bicarbonate. These investigators conducted a similar clinical trial of individuals with stage 4 CKD (eGFR 15-29 ml/ $\mathrm{min} / 1.73 \mathrm{~m}^{2}$ ) randomized to either fruits and vegetables or sodium bicarbonate for 1 year [8]. They found that the fruits and vegetables intervention reduced dietary acid load (assessed by net acid excretion and PRAL), resulting in improved metabolic acidosis status and lower concentrations of kidney injury markers. Taken together, there is growing evidence for the use of diet modification through acid load reduction in order to reduce the risk of kidney disease progression among patients with reduced eGFR.

There are several potential mechanisms to explain our finding that higher dietary acid load is associated with an increased risk of CKD. In particular, acid retention can induce the production of endothelin-1 thereby leading to kidney injury [24-27]. Acid retention can also activate the intrarenal renin-angiotensin system by stimulating production of aldosterone, which can contribute to the onset and progression of kidney disease [25, 26, 28-30]. It has been demonstrated that higher dietary acid load can result in tubular injury through elevated renal ammonium concentrations and activation of the complement pathway [31, 32].

We additionally investigated the individual components of the dietary acid load estimates. There appeared to be a significant trend across quartiles of dietary intake of phosphorus and calcium and CKD risk, but these associations were no longer statistically significant in continuous analyses. A recent systematic review found limited, low-quality evidence for the kidney effects of dietary intake of calcium and phosphorus [33]. These studies primarily assessed biochemical markers (e.g., serum concentration of calcium, phosphorus, and fibroblast growth factor-23) rather than hard clinical outcomes. An observational analysis of the Modification of Diet in Renal Disease (MDRD) study found that serum phosphorus was associated with mortality, but dietary intake of phosphorus, assessed by 24 -hour urine excretion, was not associated with mortality or any other clinical outcome [34]. It may be possible that our study was under-powered to detect these smaller effect sizes. Studies with larger sample sizes should investigate associations of dietary intake of these micronutrients with CKD risk.

We found that higher dietary intake of magnesium and vegetable sources of protein were protective against kidney disease. These 2 findings are complementary since vegetable sources of protein such as legumes are rich sources of dietary magnesium [35]. It may be that these diet factors themselves are related to kidney disease pathogenesis or that they are important contributors to the reduction of dietary acid load which is primarily responsible for the association with lower kidney disease 
risk. Intake of dietary protein from vegetable sources may protect against CKD by lowering serum concentrations of fibroblast growth factor- 23 and raising serum concentrations of bicarbonate as shown in the Chronic Renal Insufficiency Cohort (CRIC) study [36]. While clinical guidelines suggest dietary restriction of total protein for the management of kidney disease, sources of protein are not specified [18].

There are no known studies that have reported on the association between dietary intake of magnesium and incident CKD. In the ARIC Study, it has previously been shown that higher serum concentrations of magnesium, which may in part reflect dietary magnesium intake, are associated with lower risk of CKD and end-stage renal disease [37]. Further supporting the plausibility of this mechanism, low serum magnesium concentrations promote endothelial dysfunction by stimulating inflammatory and pro-atherogenic cytokines [38]. Additional studies are necessary to comprehensively investigate the health effects of dietary magnesium and replicate our finding in other cohorts.

There are a few important study limitations and strengths to be considered when interpreting these findings. Self-reported dietary intake is subject to measurement error [39, 40]. However, a previously published validation study conducted in a random subset of 419 ARIC study participants reported high reproducibility of the food frequency questionnaire, which was administered to participants by a trained interviewer [13]. In addition, we analyzed the cumulative average diet using data from 2 study visits, which has been shown to increase precision and better represent usual dietary intake [14]. Furthermore, regression models were adjusted for energy intake via the standard method in order to mitigate the impact of measurement error in dietary assessment and increase the precision of the relative risk estimate $[41,42]$. Another potential limitation is recall bias with respect to selfreported dietary intake. However, recall is not likely to be differential by CKD status given the prospective study design and use of a general population sample without CKD at baseline. Inclusion of overweight/obese status in the multivariable regression models is likely over-adjustment due to body mass index being a mediator in the relationship between dietary intake and risk of CKD. Therefore, the reported results are conservative estimates since body mass index explains part of the true association. Aside from the dietary acid load estimates, biochemical measures of acid-base status, for example, serum bicarbonate, were not available in this study. However, previous studies have established that diet can influence the acid-base status $[22,23]$. Another limitation is the lack of a measure of urine albumin as an indicator of kidney damage. As a result, some individuals with kidney damage at baseline but preserved eGFR could have been included in the analysis. We adjusted for baseline eGFR to account for variability in kidney function even among those with baseline eGFR within the normal range. In addition, some incident cases of CKD may have been missed due to the exclusion of albuminuria from the outcome definition. This limitation is balanced by the use of a validated outcome composed of clinical measures of kidney function and change in kidney function, surveillance of hospitalizations and deaths due to CKD using billing codes, and linkage to the USRDS for the identification of end-stage renal disease cases [19]. This definition of CKD differs slightly from clinical guidelines, but is appropriate for research studies to identify CKD that develops between study visits [18]. Given that the ARIC study is a large, well-characterized, prospective cohort of individuals aged 45-64 years at the time of cohort inception, these findings should be broadly generalizable to a large segment of the US population - middle-aged, AfricanAmerican and Caucasian men and women without CKD. A clinical trial to assess the effect of modifying dietary acid load on the development of incident CKD would strengthen the evidence for a causal relationship and is justified given our results in addition to previous studies.

In conclusion, higher dietary acid load is significantly related to higher risk of developing CKD in a general population sample. Within the context of few existing effective therapies for the prevention of kidney disease, this study provides evidence that dietary acid load may represent a modifiable risk factor. These findings suggest that diet modification for the reduction of acid load may prevent $\mathrm{CKD}$ in otherwise healthy individuals, which could be implemented as a cost-effective, low-risk, preventive strategy.

\section{Acknowledgments}

The ARIC study is carried out as a collaborative study supported by National Heart, Lung and Blood Institute contracts (HHSN268201100005C, HHSN268201100006C, HHSN2682011 00007C, HHSN268201100008C, HHSN268201100009C, HHSN 268201100010C, HHSN268201100011C and HHSN2682011 00012C). The authors thank the staff and participants of the ARIC study for their important contributions.

Some of the data reported here have been supplied by the USRDS. The interpretation and reporting of these data are the responsibility of the authors and in no way should be seen as an official policy or interpretation of the US government. 
Dr. M.E. Grams is supported in part by K08 DK092287 from the National Institute of Diabetes and Digestive and Kidney Diseases. Dr. D.C. Crews is supported by grant K23 DK097184 from the National Institute of Diabetes and Digestive and Kidney Diseases and the Gilbert S. Omenn Anniversary Fellowship of the Institute of Medicine.

\section{Disclosure Statement}

The authors have no relevant conflicts of interest to report.

\section{References}

1 Adeva MM, Souto G: Diet-induced metabolic acidosis. Clin Nutr 2011;30:416-421.

2 Scialla JJ, Anderson CA: Dietary acid load: a novel nutritional target in chronic kidney disease? Adv Chronic Kidney Dis 2013;20:141149.

3 Cordain L, Eaton SB, Sebastian A, Mann N, Lindeberg S, Watkins BA, O'Keefe JH, BrandMiller J: Origins and evolution of the western diet: health implications for the 21st century. Am J Clin Nutr 2005;81:341-354.

4 Coresh J, Selvin E, Stevens LA, Manzi J, Kusek JW, Eggers P, Van Lente F, Levey AS: Prevalence of chronic kidney disease in the United States. JAMA 2007;298:2038-2047.

5 Scialla JJ, Appel LJ, Astor BC, Miller ER 3rd, Beddhu S, Woodward M, Parekh RS, Anderson CA; African American Study of Kidney Disease and Hypertension Study Group: Net endogenous acid production is associated with a faster decline in GFR in African Americans. Kidney Int 2012;82:106-112.

6 Banerjee T, Crews DC, Wesson DE, Tilea AM, Saran R, Rios-Burrows N, Williams DE, Powe NR; Centers for Disease Control and Prevention Chronic Kidney Disease Surveillance Team: High dietary acid load predicts ESRD among adults with CKD. J Am Soc Nephrol 2015;26:1693-1700.

7 Goraya N, Simoni J, Jo C, Wesson DE: Dietary acid reduction with fruits and vegetables or bicarbonate attenuates kidney injury in patients with a moderately reduced glomerular filtration rate due to hypertensive nephropathy. Kidney Int 2012;81:86-93.

8 Goraya N, Simoni J, Jo CH, Wesson DE: A comparison of treating metabolic acidosis in CKD stage 4 hypertensive kidney disease with fruits and vegetables or sodium bicarbonate. Clin J Am Soc Nephrol 2013;8:371-381.

9 Sarnak MJ, Levey AS, Schoolwerth AC, Coresh J, Culleton B, Hamm LL, McCullough PA, Kasiske BL, Kelepouris E, Klag MJ, Parfrey P, Pfeffer M, Raij L, Spinosa DJ, Wilson PW; American Heart Association Councils on Kidney in Cardiovascular Disease, High Blood Pressure Research, Clinical Cardiology, and Epidemiology and Prevention: Kidney disease as a risk factor for development of cardiovascular disease: a statement from the American Heart Association Councils on Kidney in Cardiovascular Disease, High Blood Pressure Research, Clinical Cardiology, and Epidemiology and Prevention. Circulation 2003;108:2154-2169.
10 Tonelli M, Wiebe N, Culleton B, House A, Rabbat C, Fok M, McAlister F, Garg AX: Chronic kidney disease and mortality risk: a systematic review. J Am Soc Nephrol 2006;17: 2034-2047.

11 The Atherosclerosis Risk in Communities (ARIC) study: design and objectives. The ARIC investigators. Am J Epidemiol 1989; 129:687-702.

12 Willett WC, Sampson L, Stampfer MJ, Rosner B, Bain C, Witschi J, Hennekens CH, Speizer FE: Reproducibility and validity of a semiquantitative food frequency questionnaire. Am J Epidemiol 1985;122:51-65.

13 Stevens J, Metcalf PA, Dennis BH, Tell GS Shimakawa T, Folsom AR: Reliability of a food frequency questionnaire by ethnicity, gender, age and education. Nutr Res 1996;16: 735-745.

$14 \mathrm{Hu}$ FB, Stampfer MJ, Rimm E, Ascherio A, Rosner BA, Spiegelman D, Willett WC: Dietary fat and coronary heart disease: a comparison of approaches for adjusting for total energy intake and modeling repeated dietary measurements. Am J Epidemiol 1999;149: 531-540.

15 Shimakawa T, Sorlie P, Carpenter MA, Dennis B, Tell GS, Watson R, Williams OD: Dietary intake patterns and sociodemographic factors in the atherosclerosis risk in communities study. ARIC study investigators. Prev Med 1994;23:769-780.

16 Remer T, Manz F: Potential renal acid load of foods and its influence on urine $\mathrm{pH}$. J Am Diet Assoc 1995;95:791-797.

17 Frassetto LA, Todd KM, Morris RC Jr, Sebastian A: Estimation of net endogenous noncarbonic acid production in humans from diet potassium and protein contents. Am J Clin Nutr 1998;68:576-583.

18 Kidney Disease: Improving Global Outcomes (KDIGO) CKD Work Group: KDIGO clinical practice guideline for the evaluation and management of chronic kidney disease. Kidney Int Suppl 2013;3:1-150.

19 Grams ME, Rebholz CM, McMahon B, Whelton S, Ballew SH, Selvin E, Wruck L, Coresh J: Identification of incident CKD stage 3 in research studies. Am J Kidney Dis 2014;64:214221.

20 Levey AS, Stevens LA, Schmid CH, Zhang YL, Castro AF 3rd, Feldman HI, Kusek JW, Eggers P, Van Lente F, Greene T, Coresh J; CKDEPI (Chronic Kidney Disease Epidemiology
Collaboration). A new equation to estimate glomerular filtration rate. Ann Intern Med 2009;150:604-612.

21 Banerjee T, Crews DC, Wesson DE, Tilea A, Saran R, Rios Burrows N, Williams DE, Powe NR; Centers for Disease Control and Prevention Chronic Kidney Disease Surveillance Team: Dietary acid load and chronic kidney disease among adults in the United States. BMC Nephrol 2014;15:137.

22 Remer T: Influence of diet on acid-base balance. Semin Dial 2000;13:221-226.

23 Scialla JJ: The balance of the evidence on acidbase homeostasis and progression of chronic kidney disease. Kidney Int 2015;88:9-11.

24 Khanna A, Simoni J, Hacker C, Duran MJ, Wesson DE: Increased endothelin activity mediates augmented distal nephron acidification induced by dietary protein. J Am Soc Nephrol 2004;15:2266-2275.

25 Wesson DE, Simoni J: Acid retention during kidney failure induces endothelin and aldosterone production which lead to progressive GFR decline, a situation ameliorated by alkali diet. Kidney Int 2010;78:1128-1135.

26 Wesson DE, Simoni J, Broglio K, Sheather S: Acid retention accompanies reduced GFR in humans and increases plasma levels of endothelin and aldosterone. Am J Physiol Renal Physiol 2011;300:F830-F837.

27 Phisitkul S, Khanna A, Simoni J, Broglio K, Sheather S, Rajab MH, Wesson DE: Amelioration of metabolic acidosis in patients with low GFR reduced kidney endothelin production and kidney injury, and better preserved GFR. Kidney Int 2010;77:617-623.

28 Khanna A, Simoni J, Wesson DE: Endothelininduced increased aldosterone activity mediates augmented distal nephron acidification as a result of dietary protein. J Am Soc Nephrol 2005;16:1929-1935.

$29 \mathrm{Ng} \mathrm{HY}$, Chen HC, Tsai YC, Yang YK, Lee CT: Activation of intrarenal renin-angiotensin system during metabolic acidosis. Am J Nephrol 2011;34:55-63.

30 Remuzzi G, Perico N, Macia M, Ruggenenti P The role of renin-angiotensin-aldosterone system in the progression of chronic kidney disease. Kidney Int Suppl 2005;99:S57-S65.

31 Nath KA, Hostetter MK, Hostetter TH: Pathophysiology of chronic tubulo-interstitial disease in rats. Interactions of dietary acid load, ammonia, and complement component C3. J Clin Invest 1985;76:667-675. 
32 Tolins JP, Hostetter MK, Hostetter TH: Hypokalemic nephropathy in the rat. Role of ammonia in chronic tubular injury. J Clin Invest 1987;79:1447-1458.

33 Liu Z, Su G, Guo X, Wu Y, Liu X, Zou C, Zhang L, Yang Q, Xu Y, Ma W: Dietary interventions for mineral and bone disorder in people with chronic kidney disease. Cochrane Database Syst Rev 2015;9:CD010350.

34 Selamet U, Tighiouart H, Sarnak MJ, Beck G, Levey AS, Block G, Ix JH: Relationship of dietary phosphate intake with risk of end-stage renal disease and mortality in chronic kidney disease stages 3-5: the modification of diet in renal disease study. Kidney Int 2015, Epub ahead of print.

35 Boudonck KJ, Mitchell MW, Nemet L, Keresztes L, Nyska A, Shinar D, Rosenstock M: Discovery of metabolomics biomarkers for early detection of nephrotoxicity. Toxicol Pathol 2009;37:280-292.
36 Scialla JJ, Appel LJ, Wolf M, Yang W, Zhang $\mathrm{X}$, Sozio SM, Miller ER 3rd, Bazzano LA, Cuevas M, Glenn MJ, Lustigova E, Kallem RR, Porter AC, Townsend RR, Weir MR, Anderson CA; Chronic Renal Insufficiency CohortCRIC Study Group: Plant protein intake is associated with fibroblast growth factor 23 and serum bicarbonate levels in patients with chronic kidney disease: the chronic renal insufficiency cohort study. J Ren Nutr 2012;22: 379-388.e371.

37 Tin A, Grams ME, Maruthur NM, Astor BC, Couper D, Mosley TH, Selvin E, Coresh J, Kao $\mathrm{WH}$ : Results from the atherosclerosis risk in communities study suggest that low serum magnesium is associated with incident kidney disease. Kidney Int 2015;87:820-827.
38 Ferre S, Baldoli E, Leidi M, Maier JA: Magnesium deficiency promotes a pro-atherogenic phenotype in cultured human endothelial cells via activation of NFkB. Biochim Biophys Acta 2010;1802:952-958.

39 Freedman LS, Schatzkin A, Midthune D, Kipnis V: Dealing with dietary measurement error in nutritional cohort studies. J Natl Cancer Inst 2011;103:1086-1092.

40 Kipnis V, Midthune D, Freedman L, Bingham S, Day NE, Riboli E, Ferrari P, Carroll RJ: Bias in dietary-report instruments and its implications for nutritional epidemiology. Public Health Nutr 2002;5:915-923.

41 Willett WC, Howe GR, Kushi LH: Adjustment for total energy intake in epidemiologic studies. Am J Clin Nutr 1997;65(4 suppl): 1220S-1228S; discussion 1229S-1231S.

42 Willett W, Stampfer MJ: Total energy intake: implications for epidemiologic analyses. Am J Epidemiol 1986;124:17-27. 